\title{
The incidence of delirium in older people with a mood disorder is similar with lithium and valproate
}

Shulman KI, Sykora K, Gill S, et al. Incidence of delirium in older adults newly prescribed lithium or valproate: a population-based cohort study. J Clin Psychiatry 2005;66:424-7.

Q Is the incidence of delirium greater with lithium than valproate in older people with a mood disorder?

\section{METHODS}

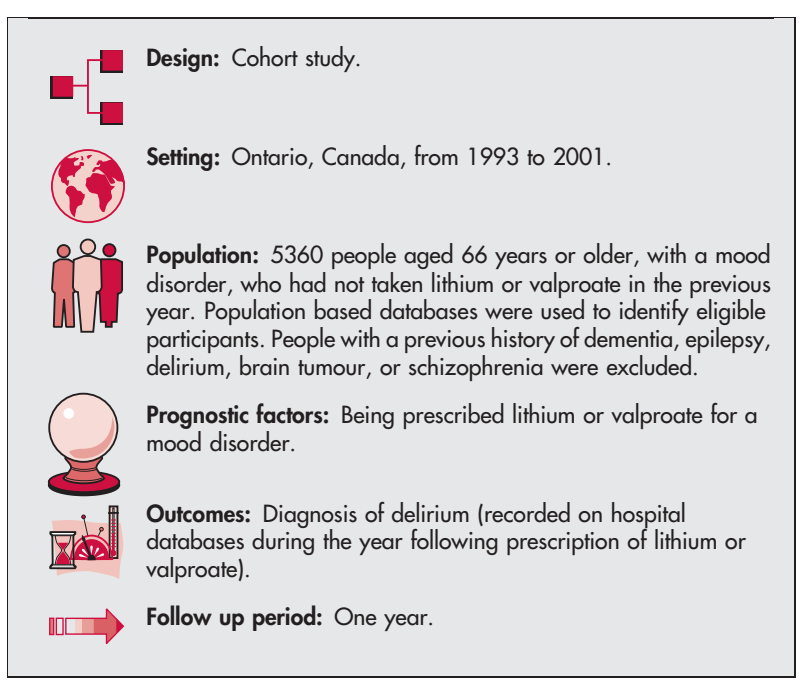

\section{MAIN RESULTS}

There was no significant difference between lithium and valproate in the incidence of delirium at one year (rate of delirium/100 person years: 4.1 with valproate $v 2.8$ with lithium; HR 1.36, 95\% CI 0.94 to 1.97).

\section{CONCLUSIONS}

There was no significant difference in the incidence of delirium between lithium and valproate in older people being treated for a mood disorder, at one year follow up.

For correspondence: Kenneth I Shulman, MD, Department of Psychiatry, Sunnybrook \& Women's College Health Sciences Centre, 2075 Bayview Avenue, Toronto, Ontario, M4N 3M5, Canada; ken.shulman@sw.ca

Source of funding: Canadian Institutes of Health Research Chronic Disease New Emerging Team Program.

\section{NOTES}

The accuracy of the findings is dependent on the accuracy of the recording of the diagnoses of delirium on hospital databases.

\section{Commentary}

D elirium as a result of psychotropic medication in the elderly can be a toxic side effect that makes caring for these patients more difficult or even medically dangerous. In recent years (as reviewed by the authors), the rate of prescribing valproate to elderly people with psychiatric illness has greatly increased, while the rate of prescription of lithium to this population has decreased. This change in prescription rates may reflect physician concern that lithium is more likely than valproate to result in delirium.

The literature on this issue is mixed, with Patten et al reporting an association between lithium and a higher risk of delirium, whereas Ritchie et al found no such increase. The current study made use of a large database with a good capture of population data derived from a single payer healthcare model in Ontario. They found that valproate and lithium did not significantly differ in the risk of incident cases of delirium, and both agents were much less likely associated with delirium than the known deliriogenic agent benztropine. Because of the other benefits of lithium, for example suicide risk reduction, avoidance of lithium in elderly mood disordered people might not be justified solely on the basis of concern of increased delirium risk.

The main clinical implications of this study, if replicated, may be that lithium (when carefully dosed and closely monitored) could be safely used in elderly people with mood disorders, in whom it may have a neuroprotective effect as well as a mood stabilisation effect. In addition, valproate may not be as free of delirium risk as previously thought. Certainly, both of these medications should be used cautiously in older people with mood disorders, with careful monitoring of serum drug levels and other laboratory parameters, and appropriate screening for delirium with acute mental status changes. Avoidance of benztropine in elderly patients on the basis of delirium risk is to be encouraged. James A Bourgeois OD MD University of California, Davis Medical Center, Sacramento, CA, USA

1 Patten SB, Williams JV, Petcu R, et al. Delirium in psychiatric inpatients: a case-control study. Can J Psychiatry 2001;46:162-6.

2 Ritchie J, Steiner W, Abrahamowicz M. Incidence of and risk factors for delirium among psychiatric inpatients. Psychiatr Serv 1996;47:727-30. 